\title{
Bolometric characteristics of macroporous silicon structures
}

\author{
L.A. Karachevtseva, O.A. Lytvynenko, E.A. Malovichko, V.D. Sobolev, O.J. Stronska \\ Institute of Semiconductor Physics, 45 Nauki Prsp., 03028 Kyiv, Ukraine \\ Tel.: 265 9815, FAX: 265 8243,E-mail:kartel@mail.kar.net
}

\begin{abstract}
Bolometric characteristics of macroporous silicon were investigated. Ohmic structures of the type «In/n-Si» and «In/macroporous $n$-Si» were formed by thermal evaporation of indium in an atmosphere of hydrogen. Temperature dependencies of electron conductivity, concentration and mobility in macroporous silicon emulate the temperature curves of the corresponding parameters for crystalline silicon. The investigated macroporous silicon structures are promising material for multi-element thermal sensor fabrication due to a high temperature coefficient of electrical resistance $(1-4 \%)$, low noise level $\left(2 \cdot 10^{-9} \mathrm{VHz}^{-1 / 2}\right)$ and good radiation absorption in the operation spectral region.
\end{abstract}

Keywords: macroporous silicon, electrochemical process, bolometric characteristics, thermal sensor.

Paper received 29.01.01; revised manuscript received 12.03.01; accepted for publication 13.07.01.

\section{Introduction}

Multi-element IR-detectors of a new type - uncooled bolometric, pyroelectric and thermoelectric matrices have been actively developed during recent years [1]. These are highly attractive for broad usage in general industry, medicine, ecological applications, transport and so on. The important characteristics required of microbolometric matrices are a high temperature coefficient of electrical resistance, low excess noise and good radiation absorption in the operation spectral region. The structures based on amorphous or polycrystalline silicon satisfy such requirements [2,3]. In this work, results of researches of macroporous silicon structures made by photoanodic etching are given. The formation of porous layers on the silicon electrodes at anodization in a solution of hydrofluoric acid has been studied since the 50s [3, 4]. The macro-, meso- and nanoporous layers were formed depending on the type of an initial substrate and characteristics of the anodic processing. The two-dimensional photonic structures based on macroporous silicon are perspective due to capability of a electromagnetic spectrum transformation by macropores. The light-assisted method of electrochemical etching of $n$-Si allows the formation of cylindrical macropores with a high ratio of pore depth to pore diameter and with high periodicity $[6$, 7]. Such structures are highly promising for modification of the electromagnetic wave spectra in semiconductor devices. Anomalous coefficients of light absorption [8] allow the development of thermal- and photo-sensors based on macroporous silicon.

Thus, the bolometric characteristics of the macroporous silicon were estimated. The ohmic indium contact to macroporous silicon structures was work up, and the temperature dependencies of conductivity, concentration and mobility of electrons, as well as spectra of excess noise were measured.

\section{Experimental}

The initial material consisted of n-type silicon with [100] orientation and $2-5 \Omega \cdot \mathrm{cm}$ resistivity. The initial $\mathrm{n}-\mathrm{Si}$ plates were chemically polished in a $1: 3 \mathrm{HF}$ and $\mathrm{HNO}_{3}$ water solution and anisotropically etched in $10 \%$ solution of $\mathrm{KOH}$ in water to allow pit formation (the periodic structures were anisotropically etched after the photolithography procedure). Ohmic contacts were provided by rubbing some In-Ga eutectic alloy on the edge of the silicon plate and by a platinum electrode in the electrolyte. The sample was mounted in an electrochemical cell and connected to a potentiostat by the 3-electrode scheme. The applied voltage was measured relatively to a platinum wire near the sample surface. The electrolyte was the 5 weight percent hydrofluoric acid in water. During 


\section{L.A. Karachevtseva et al.: Bolometric characteristics of macroporous silicon ...}

the electrochemical etching process the sample was illuminated by radiation from a $100 \mathrm{~W}$ tungsten lamp. Additional anisotropy etching in $10 \% \mathrm{KOH}$ solution was used for removing the products of the electrochemical process from the macropore walls.

Indium contacts to monocrystalline and to macroporous silicon were formed by vacuum deposition of metal and temperature annealing. Purified hydrogen was passed through palladium filters to the vacuum chamber for treatment of the structures in a gaseous atmosphere. Temperature dependencies of conductivity, concentration and mobility of electrons were measured by the 4-probe method over the temperature range 77-300 K. Excess noise was investigated in the frequency band 1$1000 \mathrm{~Hz}$ using a spectrum analyzer and a preamplifier, whose own noise in the measured frequency band was essentially less than that of a sample with $5 \mathrm{~W}$ resistance.

\section{Results and discussion}

A method of creation of an ohmic contact without an intermediate dielectric layer was realized for the formation of «metal/silicon» and «metal/macroporous silicon» low resistance contacts. With regard for the resistivity of the initial silicon substrates, metals with work function $W \leq 4.3 \mathrm{eV}$ should be used for the manufacture of an ohmic contact. This condition is satisfied by the following widely used metals: Ti $(W=3.83 \mathrm{eV}), \mathrm{Al}(W=4.18 \mathrm{eV})$ and In $(W=3.97 \mathrm{eV})$. Preference was given to In, taking into account the necessity of thermal treatment of the metal/ $\mathrm{SiO}_{\mathrm{x}} / n-\mathrm{Si}$ structure for metal diffusion through the $\mathrm{SiO}_{\mathrm{x}}$ layer and its ability to oxidation. The additional operation of cleaning the surface of the macroporous silicon samples was effected by thermal treatment in atmosphere of $\mathrm{H}_{2}$ at a pressure of $100-150 \mathrm{~mm}$ of $\mathrm{Hg}$ at $500^{\circ} \mathrm{C}$ for 30 minutes. Thermal annealing of the «In/n-Si» and «In/ macroporous $n$-Si» structures was performed at temperature of $200-500^{\circ} \mathrm{C}$.

Figs 1 and 2 show the current-voltage characteristics for «indium/silicon»s structures (Fig. 1) and for «indium/ macroporous silicon» structures (Fig. 2) measured at $300 \mathrm{~K}$. Without annealing (Figs 1a, 2a), the current-voltage characteristics of the «indium/silicon» contacts and of the «indium/macroporous silicon» contacts are

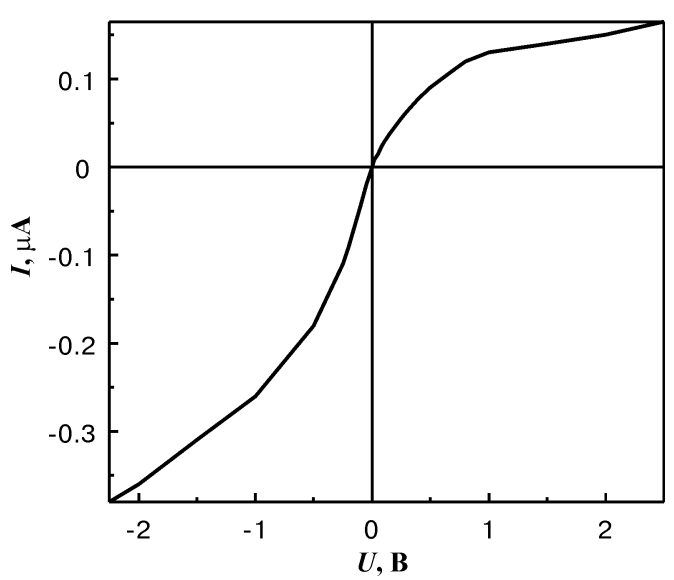

a)

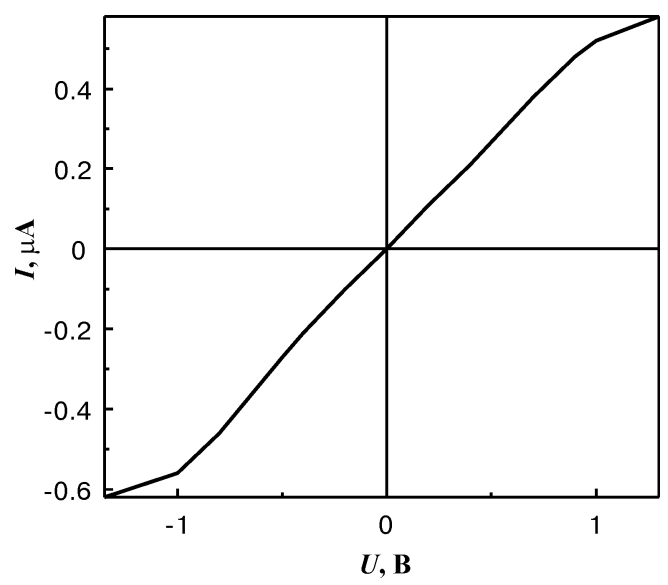

c)
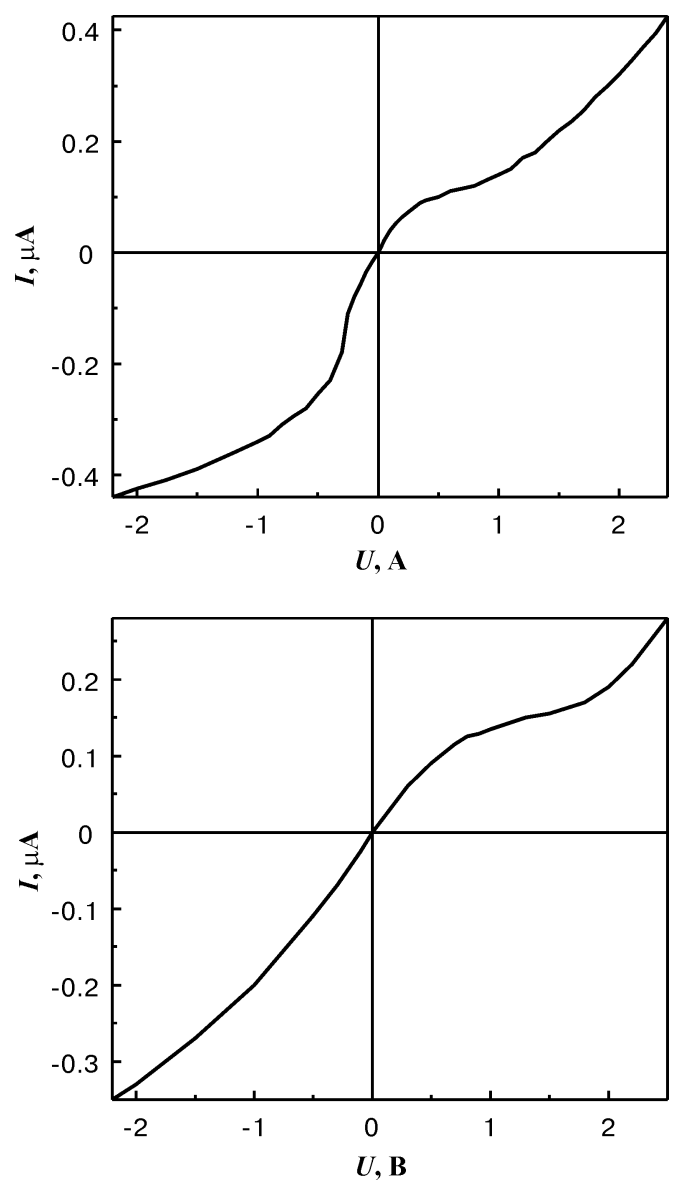

d)

Fig. 1. The current-voltage characteristics $(300 \mathrm{~K})$ for «indium/silicon» structures: (a) without annealing; (b)-(d) - at annealing temperatures 200,400 and $500^{\circ} \mathrm{C}$. 


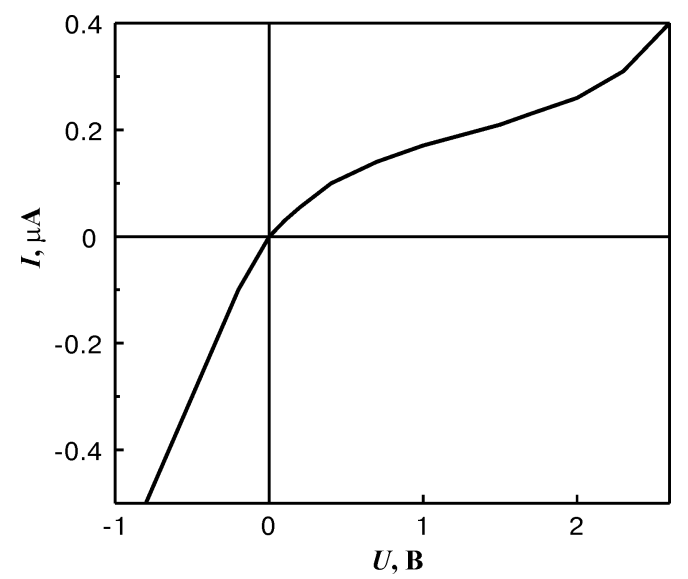

a)

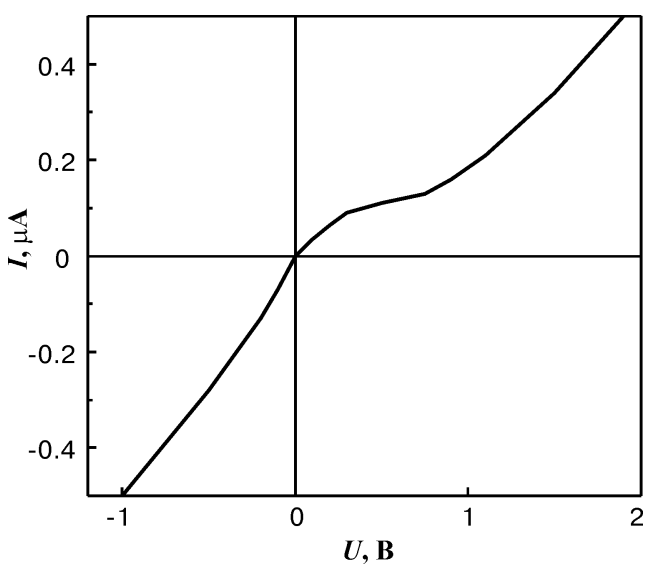

b)

c)
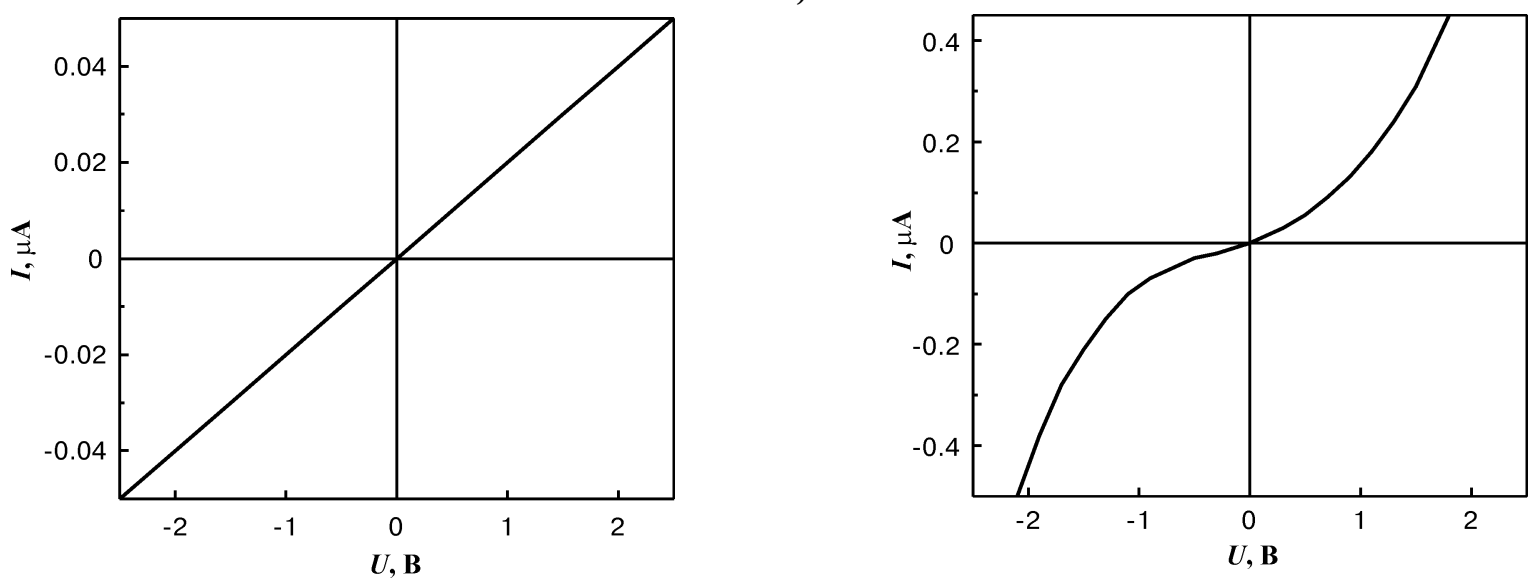

Fig. 2. The current-voltage characteristics $(300 \mathrm{~K})$ for «indium/macroporous silicon»structures: (a) without annealing; (b)-(d) - at annealing temperatures 200,400 and $500^{\circ} \mathrm{C}$.

nonlinear and are similar to the current-voltage characteristics of metal/dielectric/semiconductor structures [9]. This can be caused by an oxide layer. At higher annealing temperatures the oxide potential barrier is lowered (Figs $1 \mathrm{~b}, 2 \mathrm{~b}$ ), and at annealing temperature of $T=400^{\circ} \mathrm{C}$ in atmosphere of hydrogen the current-voltage characteristics of both structure types were linear (Figs 1c, 2c). At higher annealing temperatures the effect of overcompensation of contact area by indium took place, and the linearity of the current-voltage characteristics was broken (Figs 1d, 2d). With a change in annealing temperature from $200^{\circ} \mathrm{C}$ up to $400^{\circ} \mathrm{C}$ the transient contact resistance decreased 2 to 2.5 times and reached an optimal value of $R_{0} \approx 4-10 \Omega \cdot \mathrm{cm}^{2}$ that is less than the transient resistance of an $\mathrm{Al}$ - porous silicon contact formed at a temperature of $T=400^{\circ} \mathrm{C}$ [10].

The temperature dependencies of electron conductivity, concentration and mobility of macroporous silicon structures on $n$-Si substrates in the range 77-300 K (Fig. 3) repeat the temperature curves of the corresponding parameters for crystalline silicon. The index $p$ in the electron mobility - temperature relationship $\mu_{p} \sim T_{p}$ is high for $n$-type silicon compared with other major electronic semiconductor materials. The value $p=-2.6$ does not correspond to the theoretical value $p=-1.5$ due to various phonon scattering mechanisms. For example, twophonon scattering results in a cubic relationship $\mu_{n} \sim T^{-3}$. For impurity ionization the free charge concentration remains constant, and the temperature dependence of conductivity is determined by the temperature dependence of mobility, as in our case.

Fig. 4 shows dependencies of relative values of conductivity, concentration and mobility of electrons at temperatures $77 \mathrm{~K}$ and $300 \mathrm{~K}$ on macropore volume. The ranges of the relative temperature changes $\sigma^{77} / \sigma^{300}, n^{77} /$ $n^{300}$ and $\mu^{77} / \mu^{300}$ for macroporous silicon structures and crystal $n$-Si are comparable. The temperature coefficient of resistance ranges from $0.4-1 \%$ at room temperature to $1.5-4 \%$ at $77 \mathrm{~K}$.

Fig. 5 shows the dependencies of excess noise on frequency for the silicon substrate (a) and for macroporous silicon (b). Noise levels are comparable in the cases of initial substrate and macroporous silicon. White noise is mainly due to the Johnson one. $1 / f$ noise is sizable at frequencies less than $10 \mathrm{~Hz}$. Absolute noise values are (2-5) $\cdot 10^{-9} \mathrm{VHz}^{-1 / 2}$ for frequencies exceeding $10 \mathrm{~Hz}$, which is more than 10 times lower than the noise level in amorphous and $\alpha$-Si $[2,3]$. For an average time constant of 


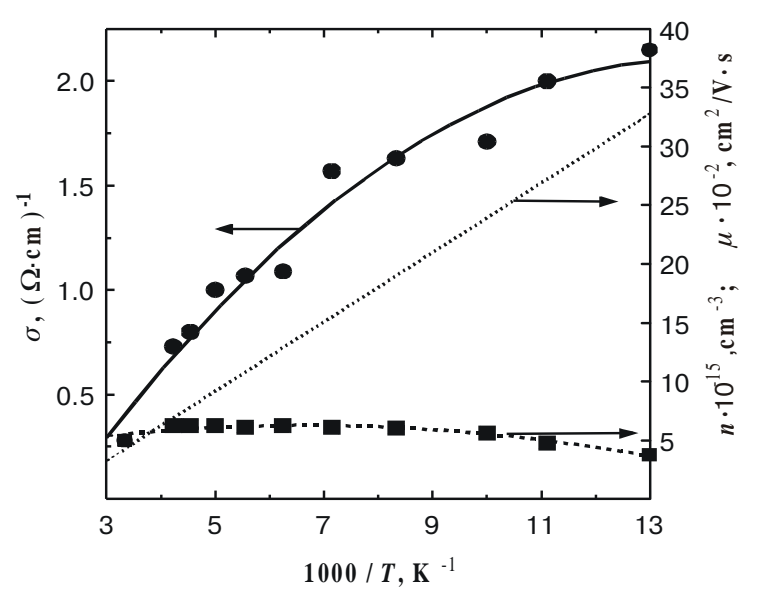

Fig. 3. The temperature dependencies of conductivity $(\bullet)$, concentration $(\bullet)$ and mobility $(\triangle)$ of macroporous silicon structures on $n$-Si substrates in the range 77-300 K.

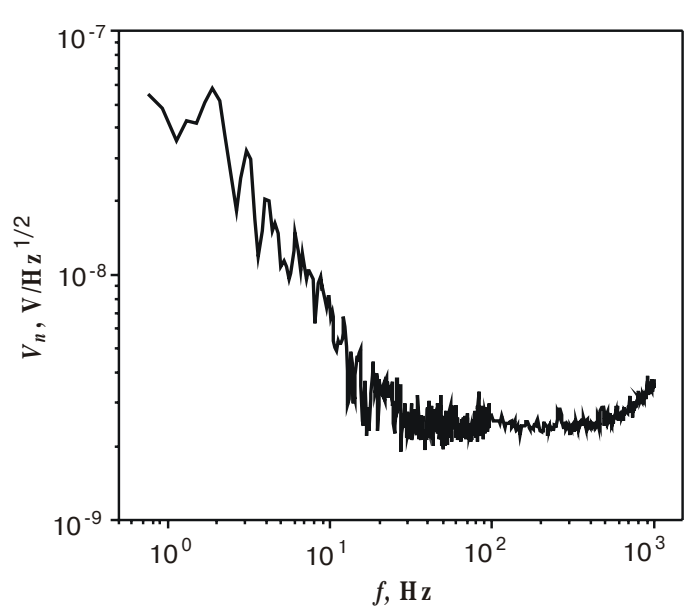

a)

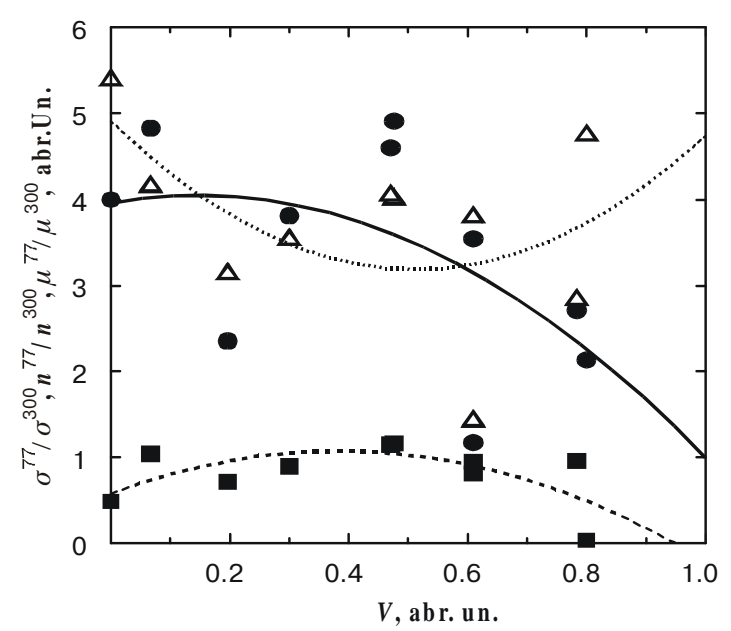

Fig. 4. Dependencies of relative values of conductivity $(\bullet)$, concentration $(\mathbf{\square})$ and mobility $(\triangle)$ of electrons at temperatures $77 \mathrm{~K}$ and $300 \mathrm{~K}$ on macropore volume.

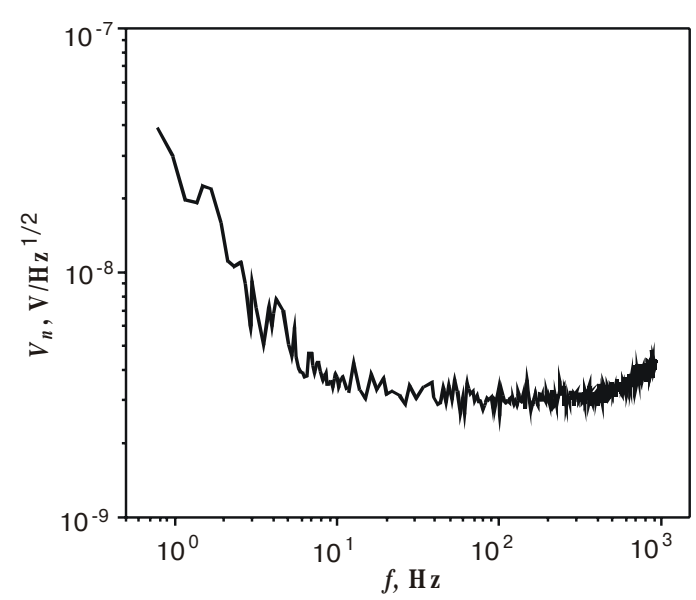

Fig. 5. Noise voltage $V_{n}$ as a function of frequency for silicon substrate with $\rho=2 \Omega \cdot \mathrm{cm}$ (a) and macroporous silicon structure (b).

$10 \mathrm{~ms}$, voltage response of $10^{3} \mathrm{~V} / \mathrm{K}$ [1], and temperature coefficient of resistance of $1 \%$, the detectivity of thermal bolometric elements based on macroporous silicon might be equal to $5 \cdot 10^{9} \mathrm{cmHz}^{1 / 2} \mathrm{~W}^{-1}$. Optical transmission of the macropore structure was only (3-6) $10^{-3}$ of apparently homogeneous material [8]. So, the investigated macroporous silicon structures are promising for multi-element thermal sensor fabrication due to a high temperature resistance coefficient, low excess noise and good radiation absorption in the operation spectral region.

\section{Conclusions}

The bolometric charateristics of macroporous silicon were estimated. Ohmic structures of the type $\mathrm{In} / n$-Si and $\mathrm{In} /$ macroporous $n$-Si were formed by thermal evaporation of indium in an atmosphere of hydrogen. Tempera- ture dependencies of electron conductivity, concentration and mobility in macroporous silicon emulate the temperature curves of the corresponding parameters for crystalline silicon. The temperature coefficient of resistance ranges from $0.4-1 \%$ at room temperature to $1.5-4 \%$ at $77 \mathrm{~K}$. Noise level is $(2-5) \cdot 10^{-9} \mathrm{VHz}^{-1 / 2}$ for frequencies exceeding $10 \mathrm{~Hz}$, and the detectivity of thermal bolometric elements based on macroporous silicon might be equal to $5 \cdot 10^{9} \mathrm{cmHz}^{1 / 2} \mathrm{~W}^{-1}$. So, the investigated macroporous silicon structures are promising for multi-element thermal sensor fabrication.

\section{References}

1. A. Rogalsky, Infrared Detectors, Electrocomponent Science Monographs, V.10, Gordon and Breach Publishers, 1999.

2. V.G. Malyarov, I.A. Khrebtov, Yu.V. Kulikov, Comparative investigations of bolometric properties of thin-film structures 


\section{L.A. Karachevtseva et al.: Bolometric characteristics of macroporous silicon ...}

based on vanadium dioxide and amorphous hydrated silicon // Applied Physics, 2, pp. 86-96 (1999) (in Russian.).

3. J. Piotrovski, L. Dobransky, Micromachined thermal devices - detectors and emitters // Opto-Electronics Rev. 6 pp. 69-78 (1998)

4. D.R. Turner, Electropolishing silicon in hydrofluoric acid solutions // J. Electrochem. Soc. 105 (7), pp. 402-408 (1958).

5. M. I. I. Theunissen, Etch channel formation during anodic dissolution of $n$-type silicon in aqueos hydrofluoric acid // J. Electrochem. Soc. 119(3), pp. 351-359 (1972).

6. V. Lehmann, H. Foll, Formation Mechanism and Properties of Electrochemically Etched Trenches in n-Type Silicon // J. Electrochem. Soc. 137(2), pp. 653-659 (1990).
7. L.A. Karachevtseva, O.A. Litvinenko, E.A. Malovichko, Stabilization of Electrochemical Formation of Macropores in $n$-Si // J. Theor. and Experim. Chem. 34 (5), pp. 314-31 (1998) (in Russian)

8. L.A. Karachevtseva, O.A. Lytvynenko, O.J. Stronska, Development and optical characteristics of the macroporous silicon structures // Semiconductor Physics, Quantum Electronics \& Optoelectronics 3(1), pp. 22-25 (2000).

9. E.H. Hroderic, Metal-Semiconductor Contacts, Clarendon, Oxford, 1978

10. S.P. Zimin, E.P. Mosquitoes, Influence of a short-term annealing on conductivity of porous silicon and transient resistance of a contact aluminium - porous silicon // J. Technical Physics Letters, 24(6), pp. 45-51 (1998) (in Russian). 